\title{
ON THE TOMOGRAPHIC INFORMATION IN SINGLE PAIRS OF CROSSING-ORBITS SAR ACQUISITIONS
}

\author{
Paco López-Dekker, Francesco De Zan, Steffen Wollstadt, Pau Prats and Gerhard Krieger \\ Microwaves and Radar Institute \\ German Aerospace Center (DLR)
}

\begin{abstract}
This paper discusses the tomographic potential of interferometric SAR acquisitions under crossing tracks, which results from the associated baseline variation. A simple model is proposed to describe the received signal. Experimental results confirm the expected tomographic potential.
\end{abstract}

Index Terms - SAR, Interferometry, Tomography

\section{INTRODUCTION}

SAR interferometry under non-parallel trajectories was first reported in 1988, in the context of the SIR-B InSAR experiments [1]. More recently, it has been reformulated and used in the first TanDEM-X single pass interferometric acquisition [2]. Since 2011 it is being used to acquire experimental 1-, 5- and 6-day repeat pass TerraSAR$\mathrm{X}$ mission acquisitions in Antarctica [3], allowing X-band DInSAR measurements in fast decorrelating areas.

SAR interferometry theory usually considers geometries in which the trajectories corresponding to the pair of InSAR acquisitions are almost perfectly parallel. A consequence of this condition is the common understanding that the two acquisitions must be acquired with similar, if not identical, Doppler centroids $\left(f_{D C}\right)$ in order to maximize the common Doppler spectral content of the images. In the case of InSAR under non-parallel trajectories, however, this common understanding is no longer valid, and an offset of the individual Doppler spectra is necessary in order to achieve interferometric coherence. This can be understood in terms of wavenumber shift theory [4]: what matters is not the overlap between the fast-time and Doppler spectra, but that of the wavenumber-domain sampling window of the ground spectrum. For crossing orbits, applying this theory leads to an intuitively appealing result: interferometric compatibility can be achieved if the SAR images are acquired with a relative squint such that the ground projections of the line-of-sight vectors (with respect to the center of the synthetic aperture) are parallel to each other [2]. The alignment of the spectra after applying a relative squint is illustrated by the right diagram in Figure 1, where all the angles are clearly exaggerated, and a zero cross-track baseline is assumed. In practice. for small rotations and squint angles, and considering that $k_{y 0} \gg 2 \pi / \delta y$, their effect is practically reduced to a spectral shift in azimuth.

Crossing-orbit InSAR acquisitions are possible at locations where the angle between two different ground-tracks of the spacecraft are small enough to be compensated by the steering capabilities of the SAR antenna, and where the cross track baseline is below the critical baseline. In practice, for sun-synchronous orbits, these requirements are very restrictive and are only fulfilled near the crossing points of these ground tracks, in the polar regions. For the 11 day repeat-cycle orbit of TerraSAR-X, the closest pairs of tracks correspond to a temporal lag of 5 or 6 days, and cross with a roughly $2^{\circ}$ angle. The second closest pairs allow a 1-day lag under a $4^{\circ}$ angle.

So far, the emphasis of these crossing orbit InSAR experiments has been on demonstrating their feasibility and on exploiting the resulting 1-, 5-, and 6-day repeat-pass acquisitions. An interesting additional feature is that, due to the relatively large crossing angle, the cross-track interferometric baseline changes rapidly in azimuth within the acquired scene. Typically, for a stripmap acquisition, this baseline variation will be of 2 to $4 \mathrm{~km}$, and it vanishes at some azimuth position. This paper focuses on studying the consequences of this baseline variation and on possible ways to exploit it. In particular, we report on a crossing-orbits Staring-Spotlight acquisitions experiment in which this extreme baseline variation occurs within the synthetic aperture.

\section{THEORY}

\subsection{Tomographic model}

It is clear that, despite the continuously varying baseline, direct beamforming based tomographic processing such as in [5] is not possible. In the case of stripmap this is because the different interferometric baselines correspond to different azimuth positions in the scene. In the case of staring spotlight acquisitions the baselines vary while the same point on the ground is being observed. However, different baselines will correspond to different azimuth wavenumbers $\left(k_{x}\right)$, which, for distributed targets, again excludes direct tomographic imaging.

An alternative approach is to invoke the Van Cittert-Zernike theorem which, in this context, establishes a Fourier transform relationship between the vertical reflectivity profile, $\eta(z)$, and the autocorrelation function of the received signal as function of the vertical wavenumber,

$$
k_{z}=2 k_{0} \frac{B_{\perp}}{r \cdot \sin \theta_{i}},
$$

where $B_{\perp}$ is the perpendicular baseline, $r$ the slant-range to the target, and $\theta_{i}$ the incident angle. Thus, for a pixel at ground-range position $y$ and azimuth position $x$, we can write

$$
\Gamma\left(k_{z}, x, y\right)=\int_{-\infty}^{\infty} \eta(z, x, y) e^{-j k_{z} z} d z
$$

From a signal theory point of view, this relation is nothing else as the Fourier transform relation between the autocorrelation function and the power spectrum for a stationary random process. The mutual coherence function $\Gamma\left(k_{z}, x, y\right)$ is the expected value of an interferogram for a given $k_{z}$ (or for a given baseline). An estimate of this 


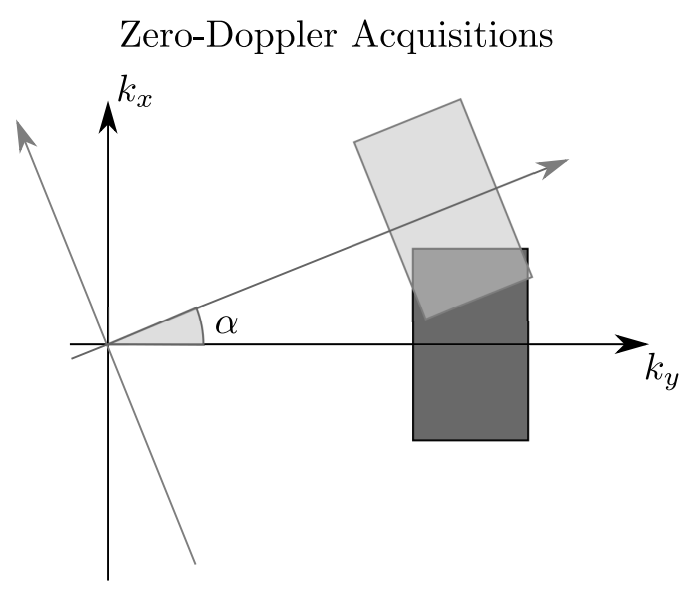

Squinted Acquisitions

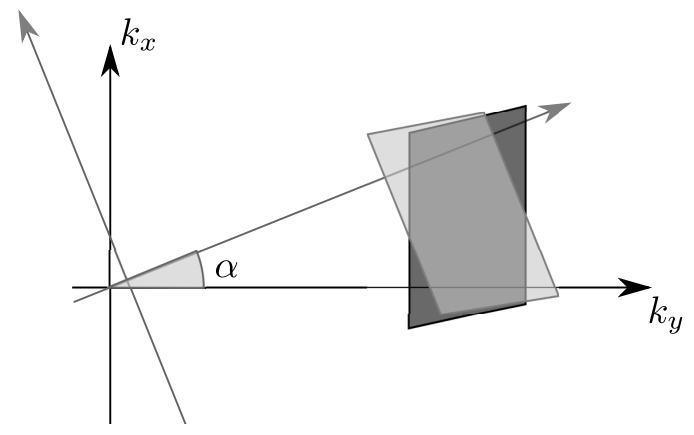

Fig. 1. Conceptual representation of the 2-D ground spectra for a pair of crossing acquisitions. Due to the rotation, if both images are acquired with $f_{D C}=0$, different parts of the 2-D ground spectrum are sampled. By acquiring the data with some relative squint, the spectral overlap is maximized.

function can be obtained by multi-looking the corresponding interferogram. Normalizing this function by the power of the SAR image results in the interferometric coherence.

Expression (2) implicitly assumes simultaneous acquisitions. However, the experiments discussed concern repeat-pass acquisitions in which a strong differential phase component, due to the motion of the ice-shelf, is present. This time-lag can be incorporated to the signal model by thinking in terms of cross-correlation functions and cross-spectra, and rewriting (2) as:

$$
\Gamma_{d}\left(k_{z}, x, y, \tau\right)=\int_{-\infty}^{\infty} \eta_{d}(z, x, y, \tau) e^{-j k_{z} z} d z
$$

Note that while $\Gamma(\cdot)$ has Hermitian symmetry with respect to $k_{z}$, which implies that only positive or negative baselines need to be sampled, this does not hold for the cross-correlation function $\Gamma_{d}(\cdot)$.

This approach to SAR tomography was already proposed in [6] for the case of of multiple single-pass TanDEM-X data-takes with varying baselines between acquisitions.

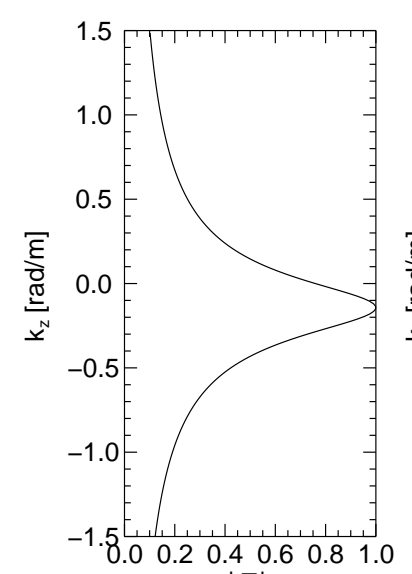

$|\Gamma|$

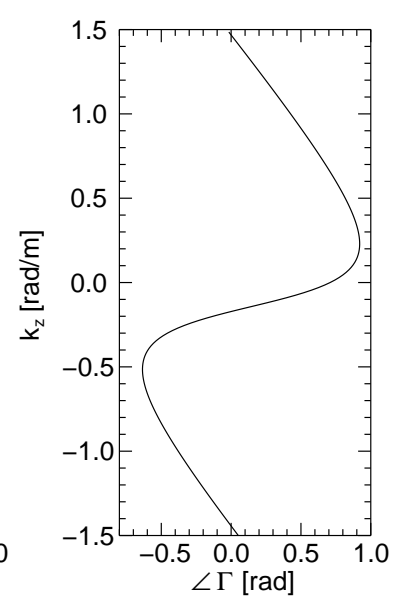

Fig. 2. Amplitude and phase of $\Gamma_{d}\left(k_{z}, \tau\right)$ according to (6) for $\delta_{z}=$ $6 \mathrm{~m}, z_{0}=1 \mathrm{~m}$, and $v_{z}^{\prime}=0.1 \mathrm{~mm} / \mathrm{m} /$ day.

\subsection{Exponential Ice Profile}

In this paper a simple vertical backscattering profile will be assumed based on an exponential decay:

$$
\eta_{d}(z, \tau)=\gamma_{t}(\tau) \frac{\sigma_{0}}{\delta_{z}} u\left(z_{0}-z\right) e^{\left(z-z_{0}\right) / \delta_{z}} e^{-j 2 k_{0} v_{r}(z) \tau},
$$

where $\gamma_{t}(\tau)$ introduces a temporal decorrelation as a function of the temporal lag $\tau, \sigma_{0}$ is the normalized radar cross section, $\delta_{z}$ the $1 / e$ penetration depth, $u(\cdot)$ the unit step function, $z_{0}$ is the height of the surface above the reference height, and $v_{r}(z)$ the vertical profile of line-of-sight velocities. Given the small penetration depth expected at X-band (in the order of $5 \mathrm{~m} \mathrm{[7]),} \mathrm{it} \mathrm{is} \mathrm{reasonable} \mathrm{to} \mathrm{linearize} \mathrm{the}$ velocity profile,

$$
v_{r}(z)=v_{r 0}+v_{r}^{\prime} \cdot\left(z-z_{0}\right)
$$

Note that since the tomographic dimension is not really vertical, but an angular dimension perpendicular to the line-of-sight, scatterers at different depths within a resolution cell will also have different horizontal positions. Thus, even in the absence of a physical vertical velocity gradient, a ground-range velocity gradient will result in a non-zero $v_{r}^{\prime}$. Substituting (4) and (5) in (3) yields

$$
\Gamma_{d}\left(k_{z}, \tau\right)=\gamma_{t}(\tau) e^{-j 2 k_{0} v_{r 0} \tau} \frac{e^{-j k_{z} z_{0}}}{1-j\left(k_{z}+2 k_{0} v_{r}^{\prime} \tau\right) \delta_{z}} .
$$

Figure 2 shows the normalized amplitude and phase of this function for an example with a penetration depth of $6 \mathrm{~m}$, a surface height of 1 $\mathrm{m}$, and a vertical gradient of the horizontal velocity of $1 \mathrm{~mm} / \mathrm{m} /$ day under a $45^{\circ}$ incident angle. An interesting and general result is that a linear vertical profile of the radial velocity results in a displacement of the cross-correlation function along the $k_{z}$ axis, so that the maximum of the interferometric coherence will not be at $k_{z}=0$ as usually expected.

\section{EXPERIMENTAL RESULTS}

The results presented in this section correspond to stripmap and staring spotlight crossing orbits acquisitions over the Ronne Ice Shelf in West-Antarctica (for a detailed description of these acquisitions 
see [3]). The observed area is extremely homogeneous and flat, and flows with a velocity in the order of $2 \mathrm{~m}$ /day predominantly from near to far range. There is also a strong gradient of this horizontal velocity in the ground range, leading to clear differential interferometric fringes.

\subsection{Stripmap acquisitions}

Due to the homogeneity of the scene, relevant tomographic information can be retrieved even for the strip-map acquisitions. The limitation in this case is that phase information needs to be discarded, since it may include an unknown topographic or differential phase. The effect of the small variations of $\sigma_{0}$ can also be avoided by analyzing the interferometric coherences. This has been done by evaluating the joint histograms of the coherence and $k_{z}$ for a series of 1-day and 5day interferograms. The theoretical coherence according to (6) can be written as

$$
\gamma\left(k_{z}\right)=\frac{\gamma_{t}(\tau)}{\sqrt{1+\delta_{z}^{2}\left(k_{z}+2 k_{0} v_{r}^{\prime} \tau\right)^{2}}}
$$

Figure 3 shows examples corresponding to acquisitions taken on April 6th, 11th and 12th, 2012. The left and right panels in the figure correspond to 5- and 1-day interferograms, respectively. The solid lines overlaid represent the average coherence for each $k_{z}$ value. A curve following (7) was fitted to these average coherences, using the maximum value of the coherence as $\gamma_{t}(\tau)$ and manually choosing $\delta_{z}$, and $v_{r}^{\prime}$. For both cases a penetration depth of $6 \mathrm{~m}$ and a vertical gradient of the horizontal velocity of $50 \mu \mathrm{m} / \mathrm{m} /$ day were chosen. It is worth emphasizing that shifts in the peak of the coherence consistent with vertical velocity gradients of this order of magnitude were found in most interferograms.

\subsection{Staring spotlight acquisitions}

In order to observe the same area with a varying baseline, a set of experimental staring spotlight acquisitions were commanded [8]. In these acquisitions, performed by the TanDEM-X satellite, the full azimuth steering range allowed by the antenna, from approximately $+2.2^{\circ}$ to $-2.2^{\circ}$, was used. Note that this is well beyond the nominal scanning range of the system, and leads to degraded azimuth ambiguity rejection. Pairs of data-takes with a 5-day lag were acquired. The results shown correspond to a pair of acquisitions taken on the April 17th and 22nd, 2012.

The images were processed and coregistered using TAXI (TanDEM-X Interferometric processor)[9] and then spectrally aligned in order to have overlapping ground spectra. Note that this step is done by simply compensating the flat-Earth phase. Due to the varying baseline, this flat Earth phase includes a strong quasi-linear azimuth phase ramp, which leads to a relative shift of the respective Doppler spectra. This shift was of approximately $12.5 \mathrm{kHz}$, or about one third of the about $40 \mathrm{kHz}$ total processed bandwidth.

After spectral alignment, the images were separated in overlapping sub-looks in the Doppler frequency domain. Each of these sublooks had roughly $1.2 \mathrm{kHz}$ bandwidth. Then, an interferogram was generated for each of these sublooks, hence constructing an estimate of $\left.\Gamma_{d}\left(k_{z}, x, y, \tau\right)\right|_{\tau=5 \text { days }}$. These estimates were subsequently flattened by estimating and subtracting the phase of the $k_{z}=0$ look.

Figure 4(a) shows the normalized amplitude and phase of estimated $\Gamma_{d}\left(k_{z}, 5\right)$, averaged over all range and azimuth pixels. The blue dashed line shows theoretical curves corresponding to a penetration depth of $8 \mathrm{~m}, v_{r}^{\prime}=30 \mu \mathrm{m} / \mathrm{m} /$ day, and a mean surface height of $5.8 \mathrm{~m}$ above the reference height.
Finally, estimates of the vertical backscattering profile were generated. In order to avoid effects due to the asymmetry in the $k_{z}$ span, Hermitian symmetry was imposed in the $k_{z}$ domain after shifting the maximum of $\left.\Gamma_{d}\left(k_{z}, x, y, \tau\right)\right|_{\tau=5}$ to zero. According to our model this step is equivalent to compensating the linear velocity gradient. Then the data were zero-padded and Fourier transformed in the $k_{z}$ dimension. Fig. 4(b) shows a vertical profile of the resulting intensity as a function of range, for the azimuth-center of the scene. As expected, the image shows a rapidly decreasing backscattering intensity below the surface.

\section{DISCUSSION}

The results presented in the previous section match with the simple theoretical model developed to describe the expected tomographic signature for our available data sets. This confirms experimentally the tomographic potential of interferometric acquisitions under crossing tracks. This tomographic potential is limited to distributed scenes with some level of homogeneity in range and in azimuth, i.e. homogeneous stratified layers are assumed within the horizontal resolution of the final product.

For repeat-pass acquisitions, the acquired data also contain differential tomographic information. Here, an advantage of the crossing-orbit acquisition strategy is that only two acquisitions are involved, which should simplify the extraction of this differential information.

An interesting observation following from the theory discussed in Section 2 is that a linear phase in the $k_{z}$ domain can be interpreted as a coregistration error in azimuth. Indeed, in the crossing orbit geometry, the master and slave images can only be coregistered correctly for scatterers at a given height. Tomographic processing can be alternatively understood as an exercise of coregistering and forming interferograms for different reference heights. This implies also that volume and surface roughness will result in local coregistration errors, and thus in a loss of coherence, even for a vanishing mean baseline.

It is clear that for SAR systems in polar orbits, crossing orbit acquisitions are limited to very particular high latitude regions. Also, the current X-band data sets are far from ideal due to the low expected penetration. Current and near future work will therefore concentrate on L-band data sets acquired with DLR's F-SAR system.

\section{REFERENCES}

[1] Andrew K. Gabriel and Richard M. Goldstein, "Crossed orbit interferometry: theory and experimental results from SIR-B," International Journal of Remote Sensing, vol. 9, no. 5, pp. 857, 1988.

[2] P. Lopez-Dekker, P. Prats, F. De Zan, D. Schulze, G. Krieger, and A. Moreira, "TanDEM-X first DEM acquisition: A crossing orbit experiment," IEEE Geoscience and Remote Sensing Letters, vol. 8, no. 5, pp. 943-947, Sept. 2011.

[3] S. Wollstadt, P. López-Dekker, P. Prats-Iraola, F. De Zan, T. Busche, and G. Krieger, "1 and 5 day differential InSAR under crossing orbits with TerraSAR-X," in Proceedings of IEEE Geoscience and Remote Sensing Symposium (IGARSS), Munich, July 2012.

[4] F. Gatelli, A. Monti Guarnieri, F. Parizzi, P. Pasquali, C. Prati, and F. Rocca, "The wavenumber shift in SAR interferometry," Geoscience and Remote Sensing, IEEE Transactions on, vol. 32, no. 4, pp. 855-865, 1994. 


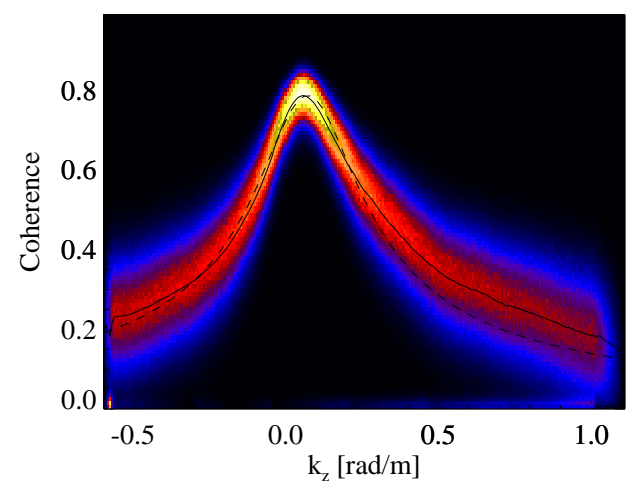

(a) 5-day
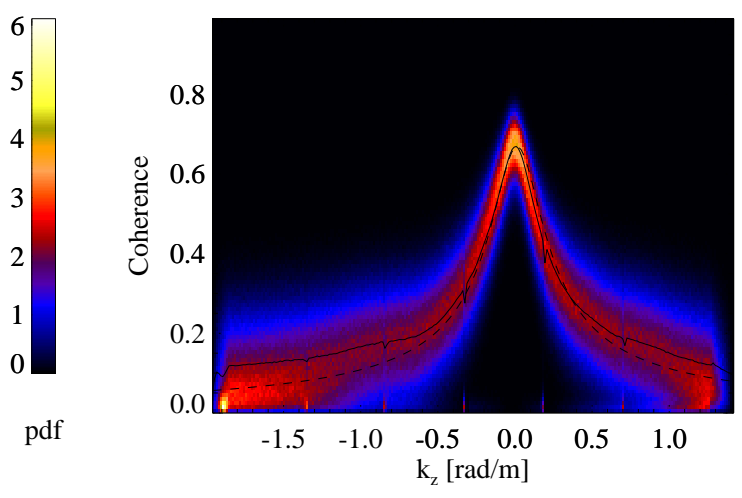

(b) 1-day

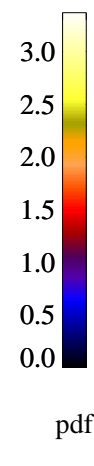

pdf

Fig. 3. Joint histograms of coherence and $k_{z}$. The left and right panels correspond, respectively, to the 5- and 1-day repeat pass interferograms acquired during April 6th, 11th and 12th, 2012. The solid lines correspond to the mean coherences as a function of $k_{z}$, while the dashed lines correspond to a manually fitted exponential decay model with $6 \mathrm{~m}$ penetration depth and a vertical velocity gradient of $50 \mu \mathrm{m} / \mathrm{m} /$ day.

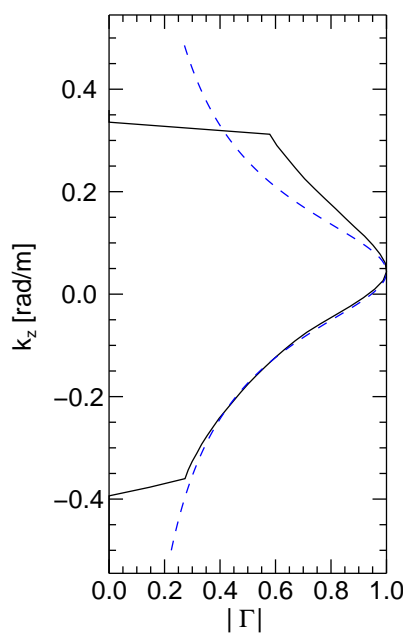

(a)

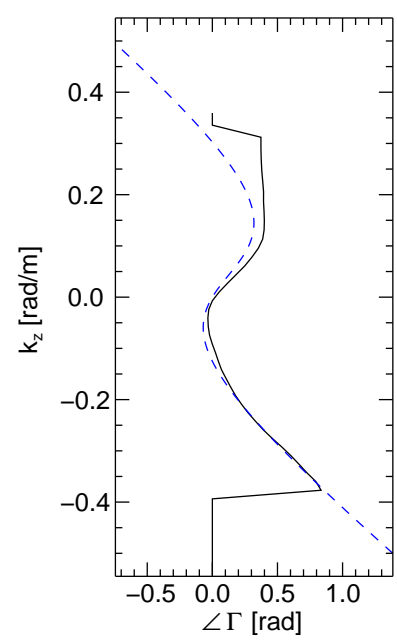

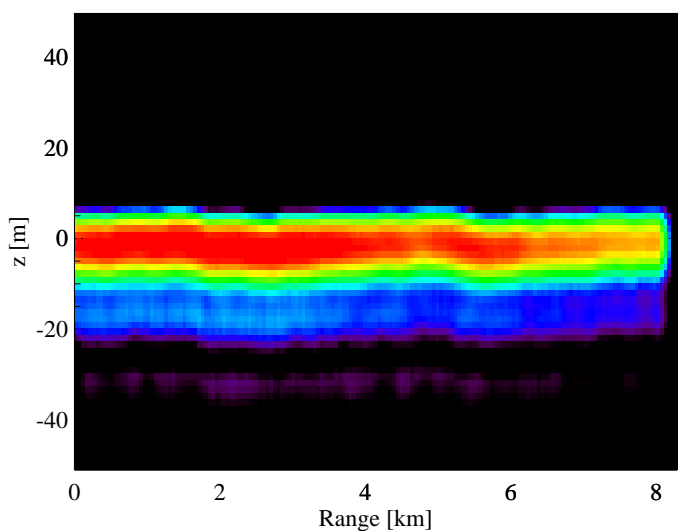

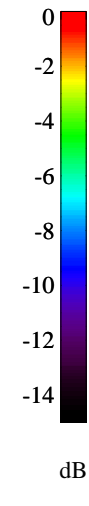

(b)

Fig. 4. Left: Normalized amplitude and phase of estimated $\Gamma_{d}\left(k_{z}, 5\right)$, averaged over all range and azimuth pixels. The blue dashed line shows theoretical curves corresponding to a penetration depth of $8 \mathrm{~m}, v_{r}^{\prime}=30 \mu \mathrm{m} / \mathrm{m} /$ day, and a mean surface height of $5.8 \mathrm{~m}$ above the reference height. Right:Vertical profile of intensity as a function of range for the azimuth-center of the acquired scene.

[5] A. Reigber and A. Moreira, "First demonstration of airborne SAR tomography using multibaseline 1-band data," IEEE Transactions on Geoscience and Remote Sensing, vol. 38, no. 5, pp. 2142-2152, Sept. 2000.

[6] G. Krieger, A. Moreira, H. Fiedler, I. Hajnsek, M. Werner, M. Younis, and M. Zink, "TanDEM-X: a satellite formation for High-Resolution SAR interferometry," Geoscience and Remote Sensing, IEEE Transactions on, vol. 45, no. 11, pp. 3317-3341, 2007.

[7] C. H Davis and V. I Poznyak, "The depth of penetration in antarctic firn at $10 \mathrm{GHz}$, IEEE Transactions on Geoscience and Remote Sensing, vol. 31, no. 5, pp. 1107-1111, Sept. 1993.

[8] J. Mittermayer, S. Wollstadt, P. Prats, R. Scheiber, and W.
Koppe, "Staring spotlight imaging with TerraSAR-X," in Proceedings of IEEE Geoscience and Remote Sensing Symposium (IGARSS), Munich, July 2012.

[9] Pau Prats, Marc Rodriguez-Cassola, Luca Marotti, Matteo Nannini, Gerhard Krieger, and Andreas Reigber, "TAXI: a versatile processing chain for experimental TanDEM-X product evaluation," in Proceedings of the International Geoscience and Remote Sensing Symposium, Honolulu, 2010. 